\title{
Diversity of polyketide synthase (PKS) genes in the metagenomic community of epilithic biofilms from the littoral zone of Lake Baikal
}

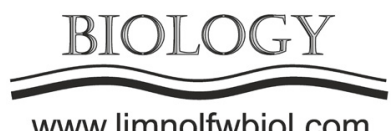

www.limnolfwbiol.com

\author{
Sukhanova E.V.*, Zimens E.A.
}

Limnological Institute, Siberian Branch of the Russian Academy of Sciences, Ulan-Batorskaya Str., 3, Irkutsk, 664033, Russia

\begin{abstract}
The secondary metabolite genes detected in the metagenomic community of epilithic biofilms of Lake Baikal belong to the representatives of well-known producers of various metabolites, e.g. Cyanobacteria, which produce toxins and antibiotics (nodularin, nostocine, microcyctine, and curacin), and Proteobacteria, which produce antibiotics (erythromycin and tuggacin). Additionally, in the production of natural substances, we detected PKS genes of new representatives: Acidobacteria, Planctomycetes and Verrucomicrobia. Most of the obtained PKS gene sequences belonged to the phylum Verrucomicrobia, which corresponds to the gene diversity typical of soil communities. The investigated biotopes are a source for the isolation and study of new producers that may possess unique active substances.
\end{abstract}

Keywords: PKS, secondary metabolites, community, biofilms

\section{Introduction}

Multi-domain enzymatic "mega-synthases", including polyketide synthases (PKSs), nonribosomal peptide synthetases (NRPSs) and their NRPS/ PKS hybrid complexes, synthesize a wide range of secondary metabolites of bacterial origin. Polyketides have a diverse chemical structure and functional activity, including antibiotics, statins, tumour growth inhibitors, and other pharmaceutically important compounds (Staunton and Wilkinson, 2001). Biofilms are complex highly dynamic structured ecosystems, in which severe chemical competition occurs; therefore, bacteria in this community must have well-equipped chemical mechanisms to be able to survive and impose themselves in such a competitive environment.

\section{Materials and methods}

During fieldworks onboard the RV "G. Titov" in August 2019, scuba divers from Limnological Institute SB RAS sampled rocky substrates from depths of 12-20 $\mathrm{m}$ in the littoral zone of Lake Baikal near the settlements of Bolshiye Koty, Listvyanka and Bolshoye Goloustnoye. The total DNA was extracted from natural samples of epilithic biofilms using phenol-chloroform extraction by standard methods (Sambrook, 2001). The resulting matrix was used in PCR with degenerate primers for PKS genes (Ehrenreich et al., 2005). The resulting amplicons were cloned in a pJET1.2/blunt vector (CloneJET PCR
Cloning Kit, Fermentas, Lithuania); then, they were transformed into the cell of the DH-5a competent $E$. coli strains. Nucleotide sequences were determined on a genetic analyser at the Syntol research and production company (Moscow, Russia). A comparative analysis of the obtained sequences was carried out using the BLASTX and BLASTP software packages.

\section{Results}

A molecular genetic analysis of the samples identified 65 amplicons that belonged to the type I PKS as well as to PKS/NRPS hybrid compounds. Moreover, the identified sequences showed similarity from 40 to $80 \%$ with the closest homologues from the NCBI database, which indicates the presence of new PKS genes in the epilithic biofilm community. The closest homologues of the detected PKS genes belonged to unclassified bacteria as well as to the phyla Cyanobacteria, Proteobacteria, Acidobacteria, Planctomycetes, and Verrucomicrobia. The bulk of the obtained sequences of PKS genes belonged to the phylum Verrucomicrobia. Among the homologous nucleotide sequences, there were genes responsible for the biosynthesis of toxins (nodularin, microcyctine, nostocine, and phthicerol) and antibiotics (tuggacin, erythromycin and curacin A). The closest homologues for PKS genes were obtained from the northern lakes of Canada, poplar phyllosphere, soil, Baikal and marine sponges. 


\section{Discussion}

In one study of meadow soils, genomes of microorganisms were obtained, in which various clusters of biosynthesis genes of polyketide and nonribosomal peptides were found (Crits-Christoph et al., 2018). These biosynthetic loci are encoded by recently identified members of Acidobacteria, Verrucomicobia and Gemmatimonadetes as well as a candidate of the phylum Rokubacteria. Bacteria of these groups are widespread in soils but were not previously associated with the production of secondary metabolites. In particular, a large number of biosynthesis genes was characterised for recently identified members of Acidobacteria, which is the most common bacterial type among soil biomes (Crits-Christoph et al., 2018). In another study, a correlation analysis between the main phyla and the diversity of A and KS domains revealed the relationship between the NRPS or PKS genes with less typical phyla, such as Bacteroidetes and Verrucomicrobia, especially in Antarctic soils (Borsetto et al., 2019). These two phyla are additional microbial constituents in the diversity of metabolites together with well-known producers: Actinobacteria, Proteobacteria, Firmicutes, and Cyanobacteria. A small number of the Verrucomicrobia genomes were analysed, and possibly new NRPS and PKS genes were detected. Unused or unstudied taxa, such as Verrucomicrobia and Bacteroidetes, are potential sources of new NRPSs and PKs (Borsetto et al., 2019). Members of another phylum, Planctomycetes, isolated from biofilms of macroalgae are promising producers of biologically active compounds because they have common characteristics, such as large genomes and complex life cycles, compared to the most biologically active bacteria, i.e. actinobacteria (Graça et al., ${ }^{2016)}$. The 13 analysed genomes of Planctomycetes showed the presence of genes or clusters of secondary metabolites. Gene screening revealed that $65 \%$ of Planctomycetes potentially have one or both secondary biologically active genes; $85 \%$ were amplified with the PKS-I primers and 55\% - with the NRPS primers (Graça et al., 2016).

\section{Conclusion}

Therefore, epilithic biofilm community of Lake Baikal contains producers of secondary metabolites with both studied characteristics (toxins and antibiotics) and new ones, i.e. this biotope is a good source for the isolation and study of new producers that may possess unique active substances.

\section{Acknowledgements}

This study was carried out under the State Task No. 0345-2016-0003 (AAAA-A16-116122110061-6) "Microbial and Viral Communities in the Biofilms of Freshwater Ecosystems: Taxonomic Diversity, Functional Characteristics, and Biotechnological Potential" and supported by the Russian Foundation of Basic Research, project No. 18-34-00443.

\section{References}

Borsetto C., Amos G.C.A., da Rocha U.N. et al. 2019. Microbial community drivers of PK/NRP gene diversity in selected global soils. Microbiome 7(78). DOI: 10.1186/ s40168-019-0692-8

Crits-Christoph A., Diamond S., Butterfield C.N. et al. 2018. Novel soil bacteria possess diverse genes for secondary metabolite biosynthesis. Nature 558(7710): 440-444. DOI: 10.1038/s41586-018-0207-y

Ehrenreich I., Waterbury J., Webb E. 2005. Distribution and diversity of natural product genes in marine and freshwater cyanobacterial cultures and genomes. Applied and Environmental Microbiology 71(11): 7401-7413. DOI: 10.1128/AEM.71.11.7401-7413.2005

Graça A.P., Calisto R., Lage O.M. 2016. Planctomycetes as novel source of bioactive molecules. Frontiers in Microbiology 7. DOI: $10.3389 /$ fmicb. 2016.01241

Sambrook J., Fritsch E.F., Maniatis T. 1989. Molecular cloning: a laboratory manual. New York: Cold Spring Harbor.

Staunton J., Wilkinson B. 2001. Combinatorial biosynthesis of polyketides and non-ribosomal peptides. Current Opinion in Chemical Biology 5: 159-164. DOI: 10.1016/S1367-5931(00)00185-X 